Open Access

\title{
Successful control of juvenile dermatomyositis-associated macrophage activation syndrome and interstitial pneumonia: distinct kinetics of interleukin- 6 and -18 levels
}

\author{
Hiroyuki Wakiguchi ${ }^{1}$, Shunji Hasegawa ${ }^{1 *}$, Reiji Hirano $^{1,2},{\text { Hidenobu Kaneyasu}{ }^{1}, \text { Midori Wakabayashi-Takahara }}^{1}$ \\ and Shouichi Ohga'
}

\begin{abstract}
Background: Macrophage activation syndrome (MAS) is the secondary hemophagocytic lymphohistiocytosis associated with rheumatic diseases. Recently, the different cytokine profiles between systemic juvenile idiopathic arthritis (sJIA)-associated MAS (sJIA-MAS) and juvenile systemic lupus erythematosus (JSLE)-associated MAS (JSLE-MAS) were reported. However, there is little information about juvenile dermatomyositis (JDM)-associated MAS (JDM-MAS).

Case presentation: A 4-year-old girl with JDM was hospitalized because of fever, erythema, hepatosplenomegaly, cytopenia, liver dysfunction and coagulopathy. Bone marrow aspiration revealed appreciable numbers of activated and hemophagocytosing macrophages. She was diagnosed as having JDM-MAS complicated with interstitial pneumonia (IP) based on the findings of the elevation of serum Krebs von den Lungen-6 (KL-6) levels and chest computed tomography findings. We analyzed circulating levels of interleukin (IL)-2,4,6,10,18, tumor necrosis factor- $a$ and interferon- $\gamma$ in the patient. Hypercytokinemia occurred at the diagnosis of MAS and IP, showing with the prominent elevations of IL-6 and IL-18 levels. The cytokine profiles were distinct from those reported in patients with sJIA-MAS or JSLE-MAS. High-dose corticosteroid and cyclosporine therapy led to a drastic improvement of MAS with decreased IL-6 levels. Subsequent cyclophosphamide therapy successfully controlled IP, paralleled with the declining pattern of IL-18 and KL-6 levels.

Conclusion: This is the first report to describe a successful treatment and the cytokine profile of JDM-MAS and IP. Serum IL-6 and IL-18 levels may be useful for predicting the disease activity of JDM-MAS and IP, respectively.
\end{abstract}

Keywords: Cytokine, Juvenile dermatomyositis, Macrophage activation syndrome, Autoimmune-associated hemophagocytic lymphohistiocytosis, Interstitial pneumonia, IL-18, IL-6, KL-6

\section{Background}

Macrophage activation syndrome (MAS) is the secondary hemophagocytic lymphohistiocytosis (HLH) associated with rheumatic diseases, which is a life-threatening complication of systemic inflammatory disorders [1]. It is clinically characterized by fever, hepatosplenomegaly, lymphadenopathy, cytopenia, intravascular coagulation

\footnotetext{
* Correspondence: shunji@yamaguchi-u.ac.jp

${ }^{1}$ Department of Pediatrics, Yamaguchi University Graduate School of Medicine, 1-1-1 Minamikogushi, Ube, Yamaguchi 755-8505, Japan Full list of author information is available at the end of the article
}

and organ failure [1]. Juvenile dermatomyositis (JDM) is a multisystem disease of uncertain origin that results in chronic inflammation of the striated muscle and skin [2]. Although MAS has been reported to develop in various rheumatic diseases, it is most often complicated in the children with systemic juvenile idiopathic arthritis (sJIA) [1]. Juvenile systemic lupus erythematosus (JSLE) is also one of the underlying rheumatic diseases in which MAS occurs more frequently seen than in the others [3]. On the other hand, MAS is a quite rare complication in JDM patients [4].

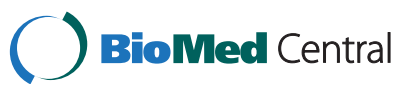

(c) 2015 Wakiguchi et al. Open Access This article is distributed under the terms of the Creative Commons Attribution 4.0 International License (http://creativecommons.org/licenses/by/4.0/), which permits unrestricted use, distribution, and reproduction in any medium, provided you give appropriate credit to the original author(s) and the source, provide a link to the Creative Commons license, and indicate if changes were made. The Creative Commons Public Domain Dedication waiver (http://creativecommons.org/publicdomain/zero/1.0/) applies to the data made available in this article, unless otherwise stated. 
Recently, the difference in the serum cytokine profiles between SJIA-associated MAS (sJIA-MAS) and JSLEassociated MAS (JSLE-MAS) was reported [5]. However, there have been no reports about the cytokine profiles in JDM-associated MAS (JDM-MAS) with interstitial pneumonia (IP). We present the first report about successful treatment and analysis of cytokine profile in a case with JDM complicated MAS and IP.

\section{Case presentation}

Case

A previously healthy 4-year-old girl exhibited butterfly like rash, heliotrope rash, Gottron's papules, mild proximal muscle weakness, muscle grasping pain, and serum creatine kinase (CK) levels and erythrocyte sedimentation rate were elevated. This patient fulfilled the diagnostic criteria for dermatomyositis [6]. To fulfill the criteria, patients must meet more than 4 findings among 8 (1. proximal muscle weakness, 2. muscle grasping and spontaneous pain, 3. nondestructive arthritis or arthralgia, 4. elevated CK or aldolase level, 5. presence of systemic inflammatory signs (fever, elevated C-reactive protein, or elevated erythrocyte sedimentation rate), 6. myogenic changes on electromyogram, 7. anti Jo-1 antibody positive, 8. pathologic findings compatible with inflammatory myositis) with having shown the characteristic skin findings (heliotrope rash or Gottron's papules). She was treated with high-dose oral prednisolone (PSL) followed by 2 courses of methylprednisolone pulse therapy (MPT). Subsequently, the skin rash and myositis gradually improved, and low-dose oral PSL was continued. Three months after MPT, she was emergently admitted to our hospital because of dyspnea, high fever, erythema, hepatosplenomegaly, cytopenia, liver dysfunction and coagulopathy. Hypoxemia, high Krebs von den Lungen-6 (KL-6) levels, and diagnostic imagings indicated progressive IP (Fig. 1a, b). Results of hematological examinations were as follows: leukocytes, $2.56 \times 10^{9} / \mathrm{L}$; hemoglobin, $13.4 \mathrm{~g} / \mathrm{dL}$; platelet count, $119 \times 10^{9} / \mathrm{L}$; D-dimer, $120.0 \mathrm{mg} / \mathrm{L}$ (reference range [rr]: $0-1 \mathrm{mg} / \mathrm{L}$ ); alanine aminotransferase (ALT), $596 \mathrm{IU} / \mathrm{L}$ (rr: 5-43 IU/L); aspartate aminotransferase (AST), 1,154 U/L (rr: 12-34 IU/L); ferritin, 8,062 ng/mL (rr: $25-280 \mathrm{ng} / \mathrm{mL}$ ); lactate dehydrogenase (LDH), 2,267 IU/L (rr: 115-217 IU/L); CK, $40 \mathrm{IU} / \mathrm{L}$ (rr: 41-123 IU/L); KL-6, 1,106 U/mL (rr: 0-499 U/dL). The urinary $\beta_{2}$-microglobulin (U- $\beta_{2}$ MG) levels were elevated, $8.06 \mathrm{mg} / \mathrm{L}(\mathrm{rr}:<0.29 \mathrm{mg} / \mathrm{L})$. The tests for antinuclear antibody (1:80; speckle) and anti-melanoma differentiation-associated gene 5 (MDA5) antibody were positive. Anti-Jo-1 antibody and Epstein-Barr virus (EBV) DNA were negative. Bone marrow aspiration showed activated macrophages phagocytosing hematopoietic elements. She was diagnosed as having JDM-MAS, and progressive IP. Heparinization, dexamethasone palmitate (DP) and cyclosporine (CsA) therapy appreciably controlled the disease activity. Six days later, the flare-up of MAS such as decrease of platelet count and elevation of serum ferritin and $U-\beta_{2}$ MG levels required MPT for 3 consecutive days, and then successfully subsided (Fig. 2). The following intravenous cyclophosphamide (IVCY) therapy led to a clinical and radiological remission of IP (Fig. 1c, d).
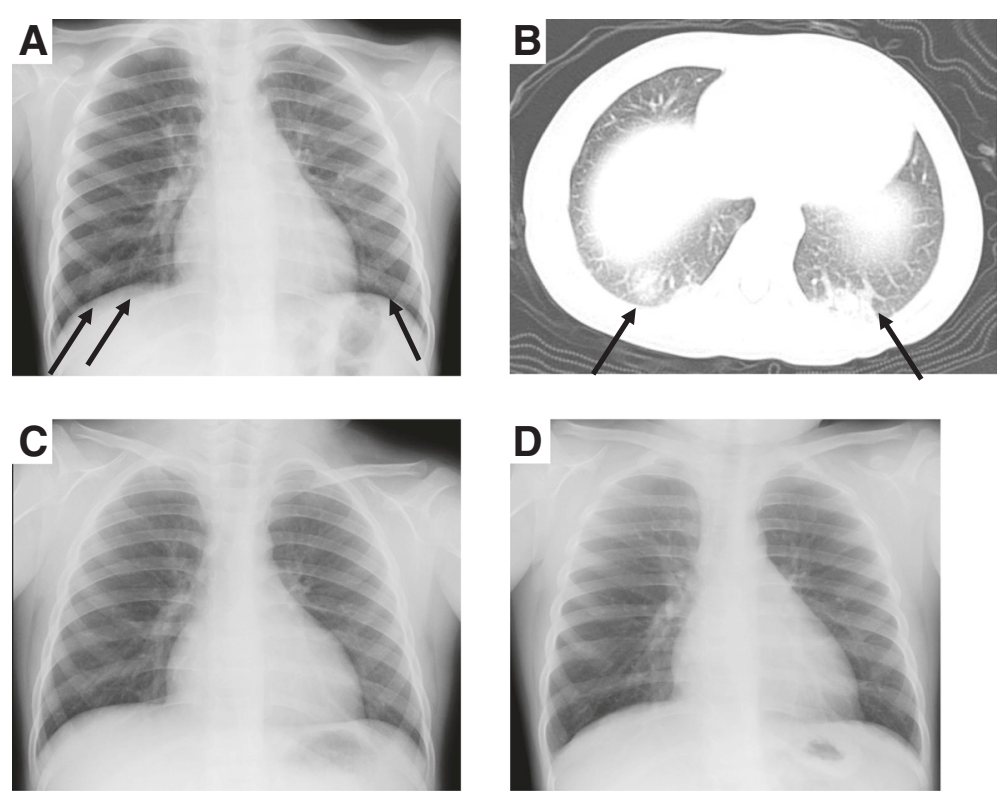

Fig. 1 Chest radiograph (a) and computed tomography (b) show focal IP in the lower lobes during the acute phase, and no abnormalities at 4 months (c) and 8 months after the convalescent phase of JDM-MAS and IP (d). Arrow marks indicate focal IP 
We studied serum cytokine profiles in this patient during the acute (days 4, 6), subacute (day 12) or convalescent phase (day 50) of JDM-MAS with IP. Multi-target streaming protein quantitative technology (BD-Pharmingen Cytometric Bead Array; BD Biosciences, Franklin Lakes, NJ, USA) was used to detect the serum cytokine levels of interleukin (IL)-2,4,6,10, tumor necrosis factor- $\alpha$ (TNF- $\alpha$ ), and interferon- $\gamma$ (IFN- $\gamma$ ), following the manufacturer's instructions. The lower detection limits for IL-2, IL-4, IL-6, IL-10, TNF- $\alpha$, and IFN- $\gamma$ were $2.6,2.6,2.5,2.8,2.8$, and $7.1 \mathrm{pg} / \mathrm{mL}$, respectively. Serum levels of IL-18 were determined using enzymelinked immunosorbent assay (ELISA) kits (Medical \& Biological Laboratories, Co., Ltd., Nagoya, Aichi, Japan) according to the manufacturer's protocols. The lower detection limit was $12.5 \mathrm{pg} / \mathrm{mL}$.

The clinical courses and the results of cytokine expressions in our patient with JDM-MAS and IP are shown in Fig. 2a, b. There was hypercytokinemia before the treatment and during the acute phase of JDM-MAS with IP.

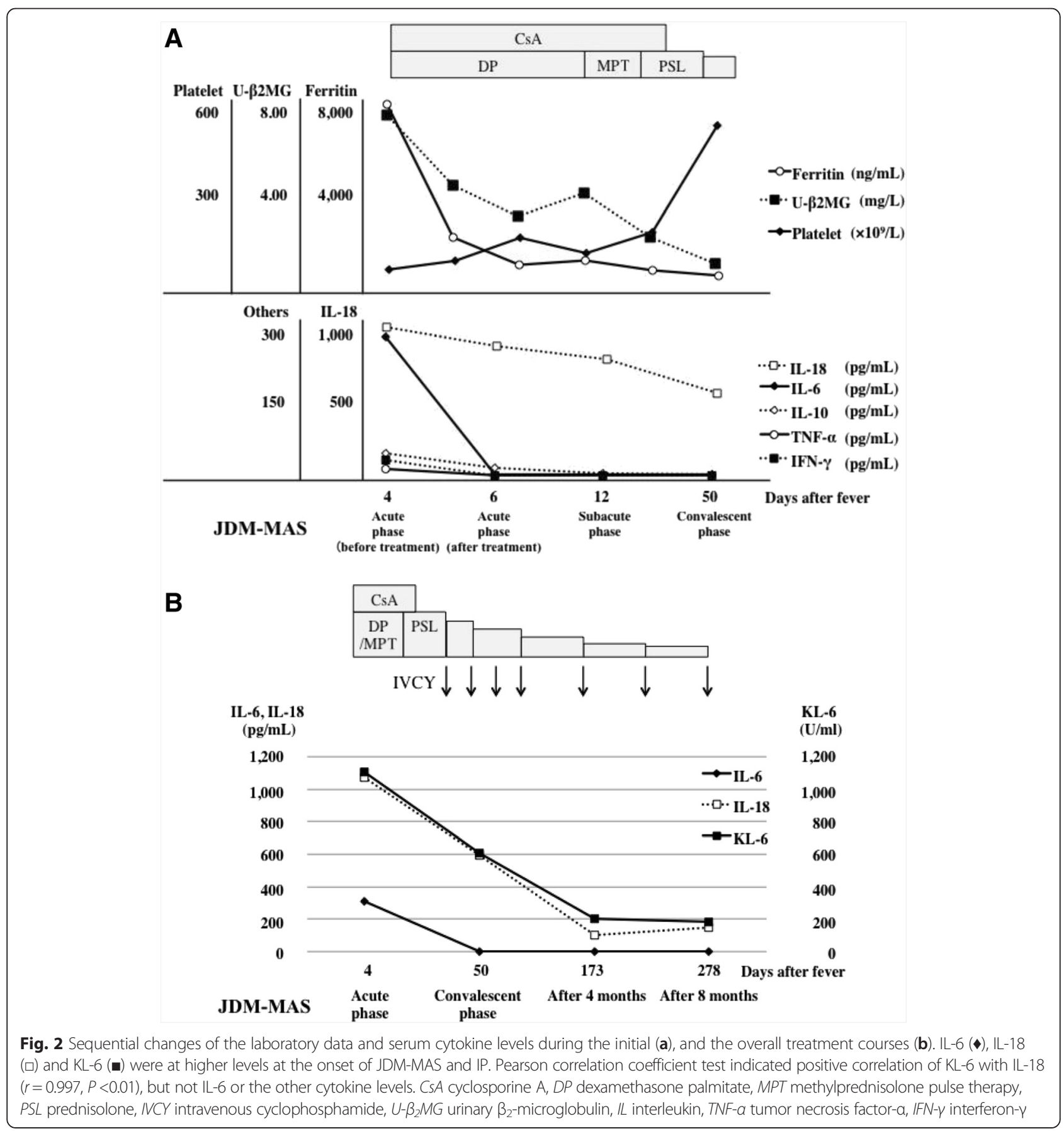


IL-6 and IL-18 were at prominent high levels compared with the elevated levels of IL-10, TNF- $\alpha$ and IFN- $\gamma$. IL-6 levels promptly decreased after the initial therapy. IL-18 levels sustained to be high after the start of effective treatments during the acute and subacute phases of JDM-MAS. On the other hand, the IL-18 levels decreased after IVCY therapy, in concert with the declining KL-6 levels and the clinical and radiographical improvement of IP (Fig. 2b).

\section{Discussion}

There has been no report about the cytokine profile in JDM-MAS with IP. To our knowledge, only 5 patients with JDM-MAS were reported [4, 7-10]. There were 4 boys and 1 girl, the median age of whom was 12 years ranging from 7 to 14 . MAS is a life-threatening condition, and the reported mortality rates reach $20 \%$ [11]. On the other hand, all 6 patients including ours survived JDM-MAS after immunosuppressive therapy. The distinct changes between IL- 6 and IL-18 levels during the treatment come of our patient may indicate the unique pathophysiology of JDM-driven complications.

Serum IL-18 concentrations in sJIA-MAS patients were reportedly higher than those seen in EBV-associated HLH (EBV-HLH) [12]. Monitoring the cytokine profile, including IL-18, may be useful for the differential diagnosis of sIIA-MAS from the other MAS/HLH. Although various proinflammatory cytokines are overexpressed in MAS patients, the prodominant elevations of TNF- $\alpha$ levels might be characteristic as shown in a patient with JSLE-MAS, compared to sJIA-MAS and EBV-HLH patients [5]. The cytokine profiles in MAS patients are considered to depend on the underlying rheumatic diseases. In the present case, both levels of IL- 6 and IL-18 were prominently increased during the acute phase of JDM-MAS with IP before treatment. The serum levels of IL-18 may be associated with the disease activity of IP, but not JDMMAS. Because Pearson correlation coefficient test indicated the positive correlation between serum KL-6 and IL-18 levels $(r=0.997, P<0.01)$ (Fig. 2b), we speculated that the leading cytokine may be IL-18 in sJIA-MAS, TNF- $\alpha$ in JSLE-MAS, and IL-6 in JDM-MAS, respectively. Serum IL-18 and TNF- $\alpha$ levels were dominantly elevated in patients with sJIA-MAS (average of 5 cases) and JSLEMAS (one case), respectively (sIIA-MAS IL-18: >100,000, TNF- $\alpha$ : 0-10, IL-6: 10-50 pg/mL, JSLE-MAS, IL-18: 1,000-3,000, TNF- $\alpha$ : > 1,000, IL-6: 100-500 pg/mL) [5, 12]. IL-18 may be dominant cytokine in sJIA-MAS compared with JSLE-MAS and JDM-MAS. In our patient with JDM-MAS, the elevation of IL-18 and TNF- $\alpha$ levels was mild, compared with sJIA-MAS and JSLE-MAS. Serum IL-6 levels may reflect the disease activity of JDM-MAS. Further studies are needed to determine the distinct cytokine profile of MAS according to the underlying disease.
Our results suggest that IL- 6 and IL-18 may play differential roles in the pathophysiology of JDM-MAS and IP, respectively. It has been reported that activated macrophages and monocytes produce proinflammatory cytokines, such as IL-6, TNF- $\alpha$ in MAS patients. On the other hand, IL-18 is produced not only from tissueresident macrophages and bone marrow macrophages, but also from dendritic cells in the muscle, and lungs in patients with JDM [13]. In the present case, there was a positive correlation between serum IL-18 and KL-6 levels (Fig. 2b). In IP complicated with JDM, IL-18 is mainly produced from activated alveolar macrophages, and KL-6 is produced from type II pneumocytes and bronchial epithelial cells during regeneration of lung tissues [13]. The kinetics of these biomarkers may represent the tissues injury and regeneration occurring in the lungs of IP patients.

\section{Conclusion}

This is the first report to describe the successful treatment and the cytokine profile in JDM-MAS with IP patient. Our results suggest that the elevations of serum IL-6 and IL-18 levels are associated with disease activity of JDM-MAS and IP, respectively. These results indicate that IL-6 and IL-18 may be useful for predicting treatment responsiveness in the pathophysiology of JDMMAS with IP. However, the limitation of the present study is a single case analyzed. Therefore, the accumulation of further case reports is necessary for identifying the pathophysiology of JDM-MAS.

\section{Consent}

Provided informed consent was obtained from the patient's guardian for publication of this case report.

\section{Abbreviations}

ALT: alanine aminotransferase; AST: aspartate aminotransferase; CsA: cyclosporine A; CK: creatine kinase; DP: dexamethasone palmitate; EBV: Epstein-Barr virus; ELISA: enzyme-linked immunosorbent assay; HLH: hemophagocytic lymphohistiocytosis;

IFN- $\gamma$ : interferon- $\gamma$; IL: interleukin; IP: interstitial pneumonia; IVCY: intravenous cyclophosphamide; JDM: juvenile dermatomyositis; JSLE: juvenile systemic lupus erythematosus; KL-6: Krebs von den Lungen-6; LDH: lactate dehydrogenase; MAS: macrophage activation syndrome; MDA5: melanoma differentiation-associated gene 5; MPT: methylprednisolone pulse therapy; PSL: prednisolone; rr: reference range; sJIA: systemic juvenile idiopathic arthritis; TNF-a: tumor necrosis factor- $\alpha_{;} U-\beta_{2} M G$ : urinary $\beta_{2}$-microglobulin.

\section{Competing interests}

The authors declare that they have no competing interests.

\section{Authors' contributions}

Wrote the paper: Wakiguchi H, Hasegawa S, Ohga S. Treated this patient: Wakiguchi H, Hirano R, Kaneyasu H. Measured cytokine levels: Hasegawa S, Wakabayashi TM. Conducted and designed research: Hasegawa S, Ohga S. All authors read and approved the final manuscript.

\section{Acknowledgements}

We thank Dr. M. Uchida and Dr. K. Fujita (Division of Pediatrics, JCHO Tokuyama Central Hospital) for the acquisition of data. 


\section{Author details}

${ }^{1}$ Department of Pediatrics, Yamaguchi University Graduate School of Medicine, 1-1-1 Minamikogushi, Ube, Yamaguchi 755-8505, Japan. ²Division of Pediatrics, Yamaguchi-ken Saiseikai Shimonoseki General Hospital, Shimonoseki, Japan.

Received: 20 April 2015 Accepted: 8 November 2015

Published online: 18 November 2015

\section{References}

1. Lin $\mathrm{Cl}$, Yu HH, Lee JH, Wang LC, Lin YT, Yang YH, et al. Clinical analysis of macrophage activation syndrome in pediatric patients with autoimmune diseases. Clin Rheumatol. 2012;31:1223-30

2. Rider LG, Lindlsy CB, Cassidy JT. Juvenile dermatomyositis. In: Cassidy JT, Petty RE, Laxer RM, Lindsley CB, editors. Textbook of pediatric rheumatology. 6th ed. Philadelphia: Saunders Elsevier; 2011. p. 375-413.

3. Ravelli A, Grom AA, Behrens EM, Cron RQ. Macrophage activation syndrome as part of systemic juvenile idiopathic arthritis: diagnosis, genetics, pathophysiology and treatment. Genes Immun. 2012;13:289-98.

4. Lilleby V, Haydon J, Sanner H, Krossness BK, Ringstad G, Flatø B. Severe macrophage activation syndrome and central nervous system involvement in juvenile dermatomyositis. Scand J Rheumatol. 2014;43:171-3.

5. Shimizu M, Yokoyama T, Tokuhisa Y, Ishikawa S, Sakakibara Y, Ueno K, et al. Distinct cytokine profile in juvenile systemic lupus erythematosus-associated macrophage activation syndrome. Clin Immunol. 2013;146:73-6.

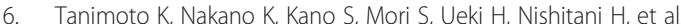
Classification criteria for polymyositis and dermatomyositis. J Rheumatol. 1995:22:668-74.

7. Kobayashi I, Yamada M, Kawamura N, Kobayashi R, Okano M, Kobayashi K. Platelet-specific hemophagocytosis in a patient with juvenile dermatomyositis. Acta Paediatr. 2000;89:617-9.

8. Sterba G, Rodriguez C, Sifontes S, Vigilanza P. Macrophage activation syndrome due to methotrexate in a 12-year-old boy with dermatomyositis. J Rheumatol. 2004;31:1014-5.

9. Kobayashi I, Kawamura N, Okano M, Sageshima S, Nakayama T, Ohyu J, et al. Thrombocytopaenia in juvenile drmatomyositis. Scand J Rheumatol. 2006; 35:79-80.

10. Bustos BR, Carrasco AC, Toledo RC. Plasmapheresis for macrophage activation syndrome and multiorgan failure as first presentation of juvenile dermatomyositis. An Pediatr (Barc). 2012;77:47-50.

11. Grom AA. Macrophage activation syndrome. In: Petty RE, Laxer RM, Lindsley CB, Wedderburn LR, editors. Textbook of pediatric rheumatology. 7th ed. Philadelphia: Saunders Elsevier; 2016. p. 642-9.

12. Shimizu M, Yokoyama T, Yamada K, Kaneda H, Wada H, Wada T, et al. Distinct cytokine profiles of systemic-onset juvenile idiopathic arthritis-associated macrophage activation syndrome with particular emphasis on the role of interleukin-18 in its pathogenesis. Rheumatology (Oxford). 2010;49:1645-53.

13. Kobayashi N, Takezaki S, Kobayashi I, Iwata N, Mori M, Nagai K, et al. Clinical and laboratory features of fatal rapidly progressive interstitial lung disease associated with juvenile dermatomyositis. Rheumatology (Oxford). 2014; online publication.

\section{Submit your next manuscript to BioMed Central and take full advantage of:}

- Convenient online submission

- Thorough peer review

- No space constraints or color figure charges

- Immediate publication on acceptance

- Inclusion in PubMed, CAS, Scopus and Google Scholar

- Research which is freely available for redistribution 